\title{
Desnutrição e desenvolvimento comportamental: questões metodológicas $^{1}$
}

\author{
Áderson Luiz Costa Junior \\ Célia Maria L. da Costa Zannon \\ Universidade de Brasilia
}

Resumo:

Este texto, de caráter introdutório, examina criticamente a relação entre desnutrição e desenvolvimento comportamental na infância, discutindo aspectos metodológicos envolvidos no estudo dessa relação. São apontadas implicações de resultados de estudos psicométricos e epidemiológicos em que se utilizam escalas cognitivas e avaliações estatísticas mais comuns. Ressalta-se a importância de tecnologia comportamental para a análise funcional do desenvolvimento de crianças desnutridas, e para o esclarecimento dos efeitos de intervenções ambientais que visam a promoção de condições favoráveis ao desenvolvimento comportamental.

Palavras-chave: Desnutrição, Comportamento, Desenvolvimento da criança, Avaliação do desenvolvimento, Tecnologia comportamental.
Key words: Malnutrition, Behavior, Child development, Developmental evaluation, Behavioral technology.

\begin{abstract}
:
Malnutrition and behavioral development: methodological issues. This introductory critical review addresses childhood malnutrition and its relation to child behavioral development. Methodological issues concerning the study of this relationship are presented. Implications of psychometric and epidemiological studies are pointed, focusing on results obtained with cognitive scales and the most common statistical measurement. The relevance of behavioral technology for functional analysis of the malnourished children development is emphasized. Its importance is also pointed for the elucidation of the effects of environmental intervention towards the establishment of favorable conditions for behavioral development.
\end{abstract}


impossível negar a influência de arranjos ambientais sobre o
curso de crescimento e de desenvolvimento comportamental comportamental é condicionado por fatores sociais e ambientais adversos e contextos caracterizados por pobreza, assim como por contextos ambientais enriquecidos (Horowitz, 1987). No caso da privação ambiental, condição de risco imposta a larga parcela da população infantil, observa-se restrição e ameaça à qualidade de vida das crianças. A desnutrição na infância, se considerados seus efeitos metabólicos, antropométricos e comportamentais, constituiria uma condição de risco (Pollitt, 1981; Pollitt \& Gorman, 1994).

No Brasil, altas taxas de mortalidade infantil podem ser efeito direto de doenças preveníveis associadas a fatores de risco de caráter socioeconômico. As condições socioeconômicas desfavoráveis de algumas regiões têm sido apontadas como "freqüentemente responsáveis por problemas de nutrição e desenvolvimento que, quando não levam à morte, produzem seqüelas que comprometem um futuro normal para estas crianças" (Fundo das Nações Unidas para a Infância [UNICEF] \& Instituto Brasileiro de Geografia e Estatística [IBGE], 1987, p. 31). Apesar de não caracterizar as "sequielas" ou o significado do termo "futuro normal", o trecho reproduzido acima afirmou a existência de uma relação entre desnutrição e comprometimento do assim denominado "curso normal de desenvolvimento" (UNICEF/IBGE, 1987, p. 31). Mais recentemente, os mesmos órgãos apontam que "existem abundantes evidências demonstrando que a desnutrição calórico-protéica nas crianças menores de 5 anos pode comprometer irreversivelmente o desenvolvimento físico e mental da criança" (UNICEF/IBGE, 1992, p. 60).

Os estudos da relação entre fatores nutricionais e ambientais sugerem variadas interpretações acerca dos efeitos da desnutrição sobre o repertório de comportamentos da criança e remetem a discussões conceituais e metodológicas.

Estudos recentes, conforme revisão de Costa Jr. (1992), mostraram que a psicologia pode dispor de metodologias comportamentais úteis ao esclarecimento da relação funcional entre a desnutrição e o 
desenvolvimento comportamental, proporcionando análises pontuais mais diretamente aplicadas ao contexto da intervenção profissional do que aquelas análises obtidas por escores e escalas de avaliação de desenvolvimento.

A literatura especializada apresenta uma série de estudos que utilizam quociente de inteligência (QI) como medida de diagnóstico de efeitos da desnutrição e de prognóstico de desenvolvimento. Fatores do ambiente relacionados à variabilidade e complexidade ambientais e à responsividade dos sujeitos a condições diferenciadas de estímulos (e.g., Yarrow, Rubenstein e Pedersen, 1975) não são suficientemente considerados quando da aplicação destes instrumentos, apesar de sya ampla utilização. A inexistência de manipulação de variáveis e a enorme amplitude dos fatores comportamentais e ambientais, não controlados nesses estudos, impedem conclusões sobre as relações funcionais entre as condições do ambiente e o comportamento dos sujeitos.

Outra dificuldade metodológica é o modo de abordar as relações entre variáveis socioeconômicas e estado nutricional, obtidas essencialmente através de estudos epidemiológicos (Shrimpton, 1986). Tais estudos são, em sua maioria, correlacionais e as relações entre estado nutricional e fatores socioeconômicos (renda, grau de instrução, condições de saneamento) são testadas em um certo ponto no tempo; as relações estabelecidas - mesmo que estatisticamente significativas - não são, necessariamente, relacionadas de modo causal.

Horowitz (1987), por exemplo, ressaltou que o nível socioeconômico é um fator geralmente definido arbitrariamente e de modo inconsistente. Mesmo que nos estudos de desenvolvimento, status socioeconômico seja freqüentemente estabelecido em termos de grau de instrução ou de ocupação dos pais, esta escolha não apresenta critérios claros que a justifiquem (pp. 180-181). Embora status socioeconômico seja reconhecido como variável relevante (em estudos que usualmente trabalham com medidas de QI), ainda não estão claros os seus componentes funcionais e a influência deste fator sobre a aquisição e manutenção do repertório de comportamentos instrumentais da criança.

Nas últimas décadas, estudos sistemáticos tentaram delinear fatores sociais e biológicos (Colombo, de Andraca \& López, 1988; 
Georgieff, 1994; Salvatierra, Castillo, Ibañez \& Mejias, 1984), determinantes do estado nutricional e do desenvolvimento biológico (Colquhoun, 1994; Lozoff, 1989; Morgane, 1993; Parker, Greer \& Zuckerman, 1988; Read, 1982; Recagno, 1982; Stein \& Susser, 1985). De modo geral, observou-se que crianças procedentes de ambientes empobrecidos sofreriam uma dupla ameaça: estariam freqüentemente expostas a riscos de caráter ambiental e sofreriam, mais do que crianças de nível socioeconômico mais elevado, as conseqüências desses riscos. Além do fato de desnutrição e pobreza ocorrerem juntas, as crianças apresentariam menor resistência a infecções e maior número de seqüelas de doenças. Ressalte-se que a correlação positiva entre desnutrição, recorrência de doenças infecciosas e baixo nível socioeconômico pode indicar um fator disposicional, mas não significa a existência de uma relação causal entre estes fatores.

Matos (1983) já recomendava uma melhor definição da condição ambiental em pesquisas sobre o desenvolvimento comportamental de crianças, uma vez que a maioria dos estudos avaliava apenas o nível de instrução e a situação econômica dos pais. Considerando os resultados desses estudos, que mostravam uma relação positiva entre nível socioeconômico dos pais e desempenho da crianças em testes, a autora chamou a atenção para a variável socioeconômica que poderia ser analisada como uma resultante de fatores diversos, como condição de alimentação, vestuário, espaço físico doméstico, mobiliário, higiene pessoal e familiar e comportamentos, tais como ausência de estímulos intelectuais e culturais à criança, baixa estimulação social e/ou pequena variabilidade da mesma. No entanto, indicadores do ambiente assim formulados não têm sido estudados, de um modo consistente, em relação a indicadores comportamentais específicos.

Há algumas variáveis que são preferencialmente estudadas quando se considera a relação entre desnutrição e repertório de comportamentos. Uma dessas variáveis é o desempenho em tarefas cognitivas. López, de Andraca e Colombo (1985) observaram diferenças no desempenho intelectual e psicomotor já na idade de 15 meses, entre crianças de classes sociais baixas e aquelas de classes média e alta. Dentro da mesma classe socioeconômica (ou seja, sem diferenças significativas no que se refere à variável socioeconômica), apontou- 
se a maior influência do nível de estimulação ambiental como fator de aceleração ou desaceleração do desenvolvimento intelectual e psicomotor da criança. No mesmo estudo, observou-se que variáveis como interação pais-criança, qualidade de ambiente social doméstico e freqüência de enfermidades devido a crenças e práticas culturais influenciaram a maior ou menor ocorrência de desnutrição infantil.

A idade dos indivíduos desnutridos também pode ser considerada um fator relevante no estudo da relação entre desnutrição e comportamento; entretanto, a análise funcional dos efeitos deste fator é dificultada pela diversidade de variáveis investigadas e pelos resultados divergentes encontrados em diferentes estudos. Alguns trabalhos, por exemplo, apontaram que efeitos funcionais e estruturais da experiência de desnutrição sobre o sistema nervoso, dependendo da idade do indivíduo, do grau e da duração da desnutrição, pareciam prejudicar a aquisição de repertório de comportamentos e o desempenho acadêmico dos sujeitos (Birch, 1985; Galler, 1985; Galler, Ramsey, Solimano \& Lowell, 1983) Outros estudos mostraram que, independente da idade da criança e da intensidade, ou duração da desnutrição, ocorreriam alterações de comportamento relacionadas à interação mãe-criança e à habilidade da criança para exploração do ambiente. Tais alterações foram indicadas através de escores inferiores em testes de desenvolvimento mental e motor e através de respostas de desatenção e diminuição de responsividade social, conforme descrito em Pollitt, Leibel e Greenfield (1981).

No entanto, Frank e Zeisel (1988) observaram que "ainda que a desnutrição produza alterações do comportamento em qualquer idade, o cérebro é particularmente vulnerável às deficiências estruturais durante o período pré-escolar" (p. 1218). McKay, Sinesterra, Mckay, Gomez e Loreda (1978) também haviam observado que a idade préescolar seria a época mais provável para o início do menor desempenho intelectual de crianças desnutridas e submetidas desde muito pequenas a um ambiente caracterizado por privações socioeconômico-culturais. De modo semelhante, Birch (1985) observou que a inadequação nutricional, nos primeiros anos, se refletiria nos anos pré-escolares; se um requisito para o desenvolvimento intelectual fosse a habilidade para processar informação e integrá-las através de sistemas sensoriais, 
tanto a desnutrição aguda quanto a subnutrição crônica poderiam contribuir para o subdesenvolvimento intelectual da criança. Em conjunto, esses estudos sugerem que a relação entre o fator idade, a desnutrição e seus possíveis efeitos sobre o desenvolvimento terá que ser estudada com um controle mais estrito das variáveis que condicionam a aquisição e o desempenho comportamental da criança

Três problemas de ordem metodológica podem ser apontados nos estudos citados, quando se avalia o desenvolvimento cognitivo de crianças desnutridas e com história de desnutrição. Primeiro, a metodologia empregada parece já supor a ocorrência de deficiências intelectuais em idades ou estágios característicos; segundo, os indivíduos podem ser alocados dentro de uma distribuição populacional e julgados em virtude da distância a uma medida de tendência central; terceiro, conforme já salientou Sidman (1986), o uso de escalas de desenvolvimento conduz o pesquisador a utilizar-se de critérios estatísticos para a definição de conceitos comportamentais, tais como responsividade, sensibilidade, variabilidade e período crítico.

Tais metodologias não têm esclarecido a função das variáveis envolvidas na relação entre desnutrição e desenvolvimento e são insuficientes em fornecer informações acerca da permanência e reversibilidade dos efeitos da desnutrição, isto é, de como se dá o desenvolvimento do indivíduo com história de desnutrição severa. Há variáveis que controlam efeitos da desnutrição comumente citados, tais como redução da atividade motora e de comportamento exploratório, apatia e irritabilidade? Que estratégias ambientais poderiam ser utilizadas para evitar, ou minimizar, a ocorrência de efeitos negativos e reabilitar o comportamento destas crianças?

Também são comuns estudos biomédicos que investigam os efeitos da desnutrição na infância. Uma limitação desses estudos é sua ênfase no crescimento físico da criança e na alimentação, em detrimento de aspectos do desenvolvimento psicológico. A Organização Mundial de Saúde [OMS] e o UNICEF estimulam a utilização de programas que incluem o acompanhamento do desenvolvimento; no entanto, têm sido priorizadas ações tais como a estimulação do aleitamento materno e a terapia de reidratação oral para redução da mortalidade em crianças. 
Embora as estatísticas comprovem a efetividade do conjunto destas estratégias, usualmente os relatórios não fazem referência a aspectos psicossociais do ambiente que estariam em efeito e que poderiam ser considerados como fontes de estimulação e experiência comportamental para promoção do desenvolvimento, melhoria da qualidade de vida e diminuição da taxa de mortalidade. Acrescente-se, ainda, que a morbidade e mortalidade infantil são índices cuja redução implica em uma queda na razão entre fatores de risco, mortes e nascimentos, não sendo consideradas variáveis específicas de qualidade de vida ou as reais condições de desenvolvimento comportamental das crianças sobreviventes.

Programas de recuperação nutricional, embora necessários, parecem ser insuficientes, se considerados isoladamente. Tem-se observado que a recuperação nutricional e a suplementação alimentar podem restaurar o crescimento físico mas não recuperam funções do desenvolvimento, debilitadas pela desnutrição (Galler, Ramsey \& Solimano, 1985; McKay \& Cols., 1978; Pollitt \& Gorman, 1994). Intervenções específicas sobre o ambiente parecem necessárias para a reabilitação do desenvolvimento, tais como visitas domiciliares e estimulação psicossocial, conforme demonstraram os estudos de Grantham-McGregor, Schofield e Powell (1987) e Super, Herrera e Mora (1990).

A ênfase na descrição detalhada de contingências ambientais se justificaria, assim, por esses e outros estudos que sugerem a relevância de intervenções psicossociais sobre o ambiente da criança, visando o estabelecimento de condições favoráveis à promoção e reabilitação comportamental de efeitos adversos da desnutrição (Brazelton, 1981; Field, McCabe \& Schneiderman, 1988; Morgane, 1993; Mullen, Coll, Vohr, Muriel \& Oh, 1988; Young, 1993; Zannon, 1981), incluindo estudos que utilizam sujeitos animais (Almeida, 1994; Crnic, 1983; Oliveira \& Almeida, 1985; Widman \& Rosellini, 1990).

Evidências apontam que certas condições ou parâmetros de variáveis do ambiente físico e social podem afetar o repertório comportamental da criança. Por exemplo, conforme apontou Crnic (1981), a magnitude dos fatores ambientais poderia ser significativa: "quando condições ambientais durante a desnutrição se aproximam 
da normalidade, os efeitos da desnutrição sobre a inteligência são minimizados" (p. 165). Entretanto, o estudo da relação entre desnutrição e desenvolvimento exige instrumentos pontuais de descrição e análise das variáveis ambientais e do repertório de comportamentos da criança. Segundo Panico-Gorayeb (1984), a mera aplicação de testes de inteligência e de escalas de desenvolvimento não parece ser a maneira mais adequada para a avaliação do desenvolvimento comportamental. Os indicadores de desenvolvimento - chamados marcos de desenvolvimento - e os procedimentos de avaliação de desempenho não estão suficientemente analisados.

A maioria dos estudos que submetem crianças desnutridas a programas de estimulação tem avaliado os resultados através de medidas de alteração de quociente de inteligência, quociente de desenvolvimento e outras medidas psicométricas (Colombo, de Andraca \& López, 1988; Escudero, Puentes, Araos, Contreras \& Latorre, 1987; Grantham-McGregor \& Buchaman, 1982; GranthamMcGregor, Schofield \& Harris, 1983; Puentes, Ibañez, Salvatierra, Mejías \& Jiménez, 1986; Victora, Barros \& Vaugham, 1988). Apesar de terem obtido escores inferiores com crianças desnutridas e comprovarem aumento destes escores após intervenção ambiental, comparando-se pré e pós-testes, estes estudos avaliaram o "nível de desenvolvimento" por grupos de sujeitos e em referência a algum critério normativo. Resultados individuais são mascarados nessas análises grupais. Além disso, ao analisar a relação entre o comportamento e as variáveis de controle, tendem a avaliar apenas o critério de produto comportamental, ignorando variáveis que poderiam interferir sobre os processos de aquisição de padrões de comportamento e estabelecimento de novas relações comportamentais.

Estudos que demonstram efeitos do arranjo de contingências sobre padrões de comportamento, conforme apontado por Zannon (1991), permitem que se observe a aquisição de repertório de comportamentos instrumentais em indivíduos desnutridos e com história de desnutrição e que se busquem procedimentos mais efetivos de intervenção ambiental; "estudos sobre sensibilidade a estímulos, plasticidade comportamental, multiplicidade instrumental e relações de equivalência, tra- 
zem elementos promissores de suporte à hipótese sobre funções dos arranjos ambientais no desenvolvimento comportamental" (p. 127).

Costa Jr. e Zannon (1992), por exemplo, em estudo que avaliou o desenvolvimento comportamental de crianças com e sem história de desnutrição severa, observaram que quando foram utilizadas medidas de desempenho em testes de inteligência todos os sujeitos com história de desnutrição apresentaram desempenho inferior aos sujeitos sem história de desnutrição; o desempenho dos sujeitos poderia estar contextualizado pelas condições de história nutricional. Assumindo que algumas tecnologias comportamentais são promissoras para a análise funcional do desenvolvimento, os autores utilizaram procedimentos de equivalência de estímulos (em que se treinavam determinadas relações complexas entre estímulos e testavam-se outras), demonstrando a possibilidade da obtenção de medidas de desenvolvimento comportamental dos sujeitos. Os resultados sugeriram que a experiência de aprendizagem sob o controle condicional de estímulos proporciona a aquisição de um repertório de comportamentos complexos, mesmo por sujeitos procedentes de ambientes socioculturais caracterizados por privação. Com medidas de aquisição e desempenho comportamental as diferenças entre os sujeitos não podem ser atribuídas à história de desnutrição, e deveriam ser analisadas sob o enfoque da história individual de aprendizagem.

Horowitz (1987) observou que a apresentação de desempenhos comportamentais novos, apropriados a situações ambientais, sugere a ocorrência de mudanças evolutivas, uma vez que pode ter sido modificada a probabilidade de relações combinatórias entre variáveis do indivíduo e do ambiente. Procedimentos tais como o utilizado por Costa Jr. e Zannon (1992) permitiram a análise de mudanças comportamentais a partir de um repertório de entrada dos sujeitos, bem como a aquisição de novas relações comportamentais sob condições controladas de estímulos. Se investigações futuras demonstrarem a generalidade deste dado, será possível afirmar que medidas comportamentais de desenvolvimento de crianças com e sem história de desnutrição podem ser obtidas e comparadas a partir dos desempenhos emergentes dos sujeitos. 


\section{Referências}

Almeida, S. S. (1994). The ontogeny of exploratory behaviors in early protein-malnourished rats exposed to the elevated plus-maze test. Psychobiology, 22, 283-288.

Birch, H. G. (1985). Malnutrition, learning, and intelligence. In M. Bloom (Org.), Life span development - Bases for preventive and interventive helping (pp. 169-172). New York: Macmillan Publishing Company.

Brazelton, T. B. (1981). Nutritional factors affecting the mother-child relationship during early infancy. In R. M. Suskind (Org.), Textbook of Pediatric Nutrition (pp. 271-283). New York: Raven Press.

Colombo, M., de Andraca, I., \& López, I. (1988). Mental development and stunding. In C. J. Waterlow (Org.). Linear growth retardation in less development countries. (Nestlé Nutrition Workshop SeriesVol. 14, pp. 201-213). New York: Raven Press.

Colquhoun, I. D. (1994). Attention deficit/hyperactive disorder: A dietary/ nutritional therapeutic approach. Care \& Education, 3, 159-172.

Costa Jr., A. L. (1992). Avaliação do desenvolvimento comportamental de crianças com e sem história de desnutrição. Dissertação de Mestrado não-publicada, Universidade de Brasília, Brasília.

Costa Jr., A. L. \& Zannon, C. M. L. C. (1992). História de desnutrição e desenvolvimento comportamental: análise metodológica de procedimentos de controle discriminativo complexo. [Resumo]. In Sociedade Brasileira de Psicologia (Org.), Resumos de Comunicações Científicas, XXII Reunião Anual de Psicologia (p. 79). Ribeirão Preto: Sociedade Brasileira de Piscoloiga.

Crnic, L. S. (1981). Separation-induced early malnutrition: Maternal, physiological and behavioral effects. Physiology \& Behavior, 26, 695-707.

Crnic, L. S. (1983). Effects of nutrition and environment on brain biochemistry and behavior. Developmental Psychobiology, 16, 129-135.

Escudero, P. A., Puentes, R. R., Araos, F. V., Contreras, A. M. D., \& Latorre, M. (1987). El hogar sustituto como sistema de recuperación integral en lactantes desnutridos y graves. Revista Chilena de Pediatria, 58, 414-418.

Field, T. M., McCabe, P. M., \& Schneiderman, N. (1988). Stress and coping across development. Hillsdale, New Jersey: Lawrence Erlbaum Associates.

Frank, D. A., \& Zeizel, S. H. (1988). Deficiência de desenvolvimento. Clínicas Pediátricas da América do Norte, 6, 1203-1227. 
Galler, J. R. (1985). Influence of early malnutrition on subsequent behavioral development. V. Child's behavior at home. Journal of the American Academy of Child Psychiatry, 24, 58-64.

Galler, J. R., Ramsey, F., Solimano, G., \& Lowell, W. (1983). The influence of early malnutrition on subsequent behavioral development. II. Classroom behavior. Journal of the American Academy of Child Psychiatry, 22, 16-22.

Georgieff, M. K. (1994). Nutritional deficiencies as developmental risk factors: Comentary on Pollitt and Gorman. In E. Pollitt \& K. S. Gorman (Orgs.), Nutritional deficiencies as developmental risk factors (pp. 145-159). Hillsdale, New Jersey: Lawrence Erlbaum Associates.

Grantham-McGregor, S., Schofield, W., \& Powell, C. (1987). Development of severely malnourished children who received psychosocial stimulation: Six-year follow-up. Pediatrics, 79, 247-254.

Grantham-McGregor, S., Schofield, W., \& Harris, L. (1983). Effects of psychosocial stimulation on mental development of severely malnourished children: An interim report.Pediatrics, 72, 239-243.

Grantham-McGregor, S. \& Buchaman, E. (1982). The development of an adopted child recovering form severe malnutrition. Human Nutrition: Clinical Nutrition, 36, 251-256.

Horowitz, F. D. (1987). Exploring developmental theories: Toward a structural / behavioral model of development. Hillsdale, New Jersey: Lawrence Erlbaum Associates.

López, I., de Andraca, I., \& Colombo, M. (1985). Relevance of psychological rehabilitation in severe malnutrition. Annales Nestlé, $43,31-41$.

Lozoff, B. (1989). Nutrition and behavior. American Psychologist, 44, 231-236.

Matos, M. A. (1983). Ambiente e desenvolvimento infantil. Pediatria Moderna, 28, 283-288.

McKay, H., Sinesterra, L., McKay, A., Gomez, H., \& Loreda, P. (1978). Improving preschool cognitive ability in chronically deprived children. Science, 200, 270-278.

Morgane, P.J. (1993). Prenatal malnutrition and development of brain. Neuroscience \& Biobehavioral Reviews, 17, 91-128.

Mullen, M.K., Coll, C. G., Vohr, B. R., \& Oh, W. (1988). Motherinfant feeding interaction in full-term small-for-gestational-age infants. The Journal of Pediatrics, 112, 143-148.

Oliveira, L. M. \& Almeida, S. S. (1985). Effects of malnutrition and environment of the acquisition and extinction of avoidance behavior in rats. Physiology \& Behavior, 34, 141-145. 
Panico-Gorayeb, S. R. (1984). Descrição do repertório comportamental de crianças de um a seis meses de idade: elaboração de instrumentos e sua aplicação no estudo do desenvolvimento infantil. Tese de Doutorado não-publicada, Universidade de São Paulo, São Paulo.

Parker, S. ,Greer, S., \& Zuckerman, B. (1988). Duplo perigo: o impacto da pobreza no desenvolvimento inicial da criança. Clínicas Pediátricas da América do Norte, 6, 1253-1268.

Pollitt, E. \& Gorman, K. S. (1994). Nutritional deficiencies as developmental risk factors. Hillsdale, N. J.: Lawrence Erlbaum Associates.

Pollitt, E. (1981). Effects of nutritional supplementation on the behavioral development of infants and children. In R.M. Suskind (Org.), Textbook of Pediatric Nutrition (pp. 263-283). New York: Raven Press.

Pollitt, E., Leibel, R., \& Greenfield, D. (1981). Brief fasting, stress, and cognition in children. American Journal of Clinical Nutrition, 34, 1526-1533.

Puentes, R. R., Ibañez, S. T., Salvatierra, V. F., Mejías, M. V. C., \& Jimenez, A. A. M. (1986). Tratamiento ambulatorio de la desnutrición calórico-proteica del lactante. Consultorio de nutrición infantil. Revista Chilena de Pediatria, 57, 91-96.

Read, M. S. (1982). Malnutrition and behavior. Applied Research in Mental Retardation, 3, 279-291.

Recagno, I. (1982). Hábitos de crianza y marginalidad. Caracas: Universidad Central de Venezuela.

Salvatierra,V., Castillo, C., Ibañez, S.T., \& Mejías, M. C. C. (1984). Evaluación de un programa ambulatorio de estimulación del desarrollo psicomotor en lactantes desnutridos. Revista Chilena de Pediatria, 55, 47-53.

Sidman, M. (1986). The measurement of behavioral development. In N. A. Krasnegor, D. B. Gray, \& T. Thompson (Orgs.), Advances in Behavioral Pharmacology, (Vol. 5 - Developmental behavioral pharmacology, pp. 43-52). Hillslade, New Jersey: Lawrence Erlbaum Associates.

Shrimpton, R. (1986). Ecologia da desnutrição na infância: análise da evidência das relações entre variáveis socioeconômicas $e$ estado nutricional. Brasília: UNICEF / CNRH/IPEA.

Stein, Z., \& Susser, M. (1985). Effects of early nutrition on neurological and mental competence in human beings. Psychological Medicine, $15,717-726$.

Super, C. M., Herrera, M. G., \& Mora, J. O. (1990). Long-term effects of food supplementation and psychosocial intervention on the 
physical growth of Colombian infants at risk of malnutrition. Child Development, 61, 29-49.

UNICEF/IBGE. (1987). Crianças \& Adolescentes - Indicadores sociais, 1, 23-35.

UNICEF/IBGE. (1992). Crianças \& Adolescentes - Indicadores sociais, 4, 35-94.

Victora, C. G., Barros, F. C., \& Vaughan, J. P. (1988), Epidemiologia da desigualdade. São Paulo: HUCITEC.

Yarrow, L. J., Rubenstein, J. L., \& Pedersen, F. A. (1975). Infant and environment - Early cognitive and motivational development. New York: John Wiley \& Sons.

Young, S. N. (1993). Nutrition as a contributing factor in psychiatric disorders and their treatment. New York: Springer Publishing Company.

Widman, D. R. \& Rosellini, R. A. (1990). Restricted daily exposure to environmental enrichment increases the diversity of exploration. Physiology \& Behavior, 47, 57-62.

Zannon, C. M. L. C. (1981). O comportamento de crianças hospitalizadas e a rotina hospitalar: subsídios para atuação do psicólogo junto à equipe de Pediatria. Tese de Doutorado nãopublicada. Universidade de São Paulo, São Paulo.

Zannon, C. M. L. C. (1991). Desenvolvimento psicológico da criança: questões básicas relevantes à intervenção comportamental no ambiente hospitalar. Psicologia: Teoria e Pesquisa, 7, 119-136.

\begin{tabular}{l|ll} 
Nota & ${ }^{1}$ Texto baseado, parcialmente, em estudo
\end{tabular} de dissertação de mestrado do primeiro autor sob orientação do segundo, apresentado ao Instituto de Psicologia da Universidade de Brasília, em agosto de 
276 A. L. Costa Jr. e C. M. L. C. Zannon

1992. Trabalho desenvolvido com bolsa de mestrado do CNPq para o primeiro autor.

2 Desenvolvimento comportamental é entendido "como o estabelecimento e a mudança de padrões de relação comportamental indivíduo-ambiente, no decorrer do ciclo vital" (cf. Zannon, 1991, p. 122).

Áderson Luiz Costa Junior é professor do Instituto de Psicologia da Universidade de Brasília. Mestre em Psicologia pela Universidade de Brasília. Célia Maria Lana da Costa Zannon é professora do Instituto de Psicologia da Universidade de Brasília. Doutora em Psicologia pela Universidade de São Paulo. Endereço para correspondência: Universidade de Brasília, Instituto de Psicologia, Departamento de Psicologia Escolar e do Desenvolvimento, 70910-900 Brasília D. F. E-mail: aderson@unb.br zannon@unb.br.
Sobre o autor 